University of Nebraska - Lincoln

DigitalCommons@University of Nebraska - Lincoln

Xia Hong Publications

Research Papers in Physics and Astronomy

2010

Unusual resistance hysteresis in n-layer graphene field effect transistors fabricated on ferroelectric $\mathrm{Pb}(\mathrm{Zr} 0.2 \mathrm{Ti0} .8) \mathrm{O} 3$

X. Hong

J.Hoffman

A. Posadas

K.Zou

C. H. Ahn

See next page for additional authors

Follow this and additional works at: https://digitalcommons.unl.edu/physicshong

Part of the Atomic, Molecular and Optical Physics Commons, and the Engineering Physics Commons

This Article is brought to you for free and open access by the Research Papers in Physics and Astronomy at DigitalCommons@University of Nebraska Lincoln. It has been accepted for inclusion in Xia Hong Publications by an authorized administrator of DigitalCommons@University of Nebraska Lincoln. 
Authors

X. Hong, J. Hoffman, A. Posadas, K. Zou, C. H. Ahn, and J. Zhu 


\title{
Unusual resistance hysteresis in $n$-layer graphene field effect transistors fabricated on ferroelectric $\mathrm{Pb}\left(\mathrm{Zr}_{0.2} \mathrm{Ti}_{0.8}\right) \mathrm{O}_{3}$
}

\author{
X. Hong, ${ }^{1, a)}$ J. Hoffman, ${ }^{2}$ A. Posadas, ${ }^{2}$ K. Zou, ${ }^{1}$ C. H. Ahn, ${ }^{2}$ and J. Zhu ${ }^{1}$ \\ ${ }^{1}$ Department of Physics, The Pennsylvania State University, University Park, Pennsylvania 16802, USA \\ ${ }^{2}$ Department of Applied Physics, Yale University, New Haven, Connecticut 06520, USA
}

(Received 27 May 2010; accepted 1 July 2010; published online 23 July 2010)

\begin{abstract}
We have fabricated $n$-layer graphene field effect transistors on epitaxial ferroelectric $\mathrm{Pb}\left(\mathrm{Zr}_{0.2} \mathrm{Ti}_{0.8}\right) \mathrm{O}_{3}(\mathrm{PZT})$ thin films. At low gate voltages, PZT behaves as a high- $\kappa$ dielectric with $\kappa$ up to 100. An unusual resistance hysteresis occurs in gate sweeps at high voltages, with its direction opposite to that expected from the polarization switching of PZT. The relaxation of the metastable state is thermally activated, with an activation barrier of 50-110 meV and a time constant of $6 \mathrm{~h}$ at $300 \mathrm{~K}$. We attribute its origin to the slow dissociation/recombination dynamics of water molecules adsorbed at the graphene-PZT interface. This robust hysteresis can potentially be used to construct graphene-ferroelectric hybrid memory devices. () 2010 American Institute of Physics.

[doi:10.1063/1.3467450]
\end{abstract}

The intrinsic, unusual electronic properties of graphene have made it a promising material for developing carbon-electronics, ${ }^{1}$ such as rf-transistors ${ }^{2}$ and spin transport devices. ${ }^{3,4}$ Additional functionalities may be introduced by integrating graphene with functional oxides such as ferroelectric $\mathrm{Pb}(\mathrm{Zr}, \mathrm{Ti}) \mathrm{O}_{3}(\mathrm{PZT})$, which can offer high-efficiency gating, local density modulation, and memory functions through a spontaneous polarization that is large, electric field switchable, and nonvolatile. Integration of ferroelectrics with nanomaterials has previously been explored in nanowires ${ }^{5}$ and carbon nanotubes ${ }^{6}$ using crystalline films, and recently in graphene using a polymer layer ${ }^{7}$ as the gate dielectric. In a previous study, we demonstrated high-efficiency carrier injection and superb electron mobility up to $140000 \mathrm{~cm}^{2} / \mathrm{V} \mathrm{s}$ at low temperature in graphene transistors fabricated on high-quality epitaxial PZT films. ${ }^{8}$

In this study, we report the observation of unusual resistance hysteresis in PZT-gated high mobility $n$-layer graphene $(n$-LG) field effect transistors (FETs). The metastable resistance states exhibit relaxation times of $\sim 6 \mathrm{~h}$ at $300 \mathrm{~K}$ and 80 days at $77 \mathrm{~K}$, which is promising for constructing graphene-ferroelectric hybrid memories. The direction of the hysteresis and its thermally activated relaxation point to slow dynamics of graphene/PZT interfacial adsorbates as its origin.

We grow highly crystalline and smooth epitaxial PZT films on Nb-doped single-crystal $\mathrm{SrTiO}_{3}$ substrates. ${ }^{9}$ Characterization details are given in the supplementary material. ${ }^{10}$ Piezoresponse force microscopy experiments show the asgrown polarization of the film, $P$, points uniformly toward the substrate. To reverse $P$, a negative voltage of $8 \mathrm{~V}$ relative to the substrate is required. ${ }^{10,11}$ Graphene sheets are mechanically exfoliated on PZT and identified optically [Fig. 1(a) inset]. Thin sheets are examined with atomic force microscopy (AFM) and Raman spectroscopy. Figure 1(a) shows the AFM image of a multilayer sheet, with the parts of 1,2, 3 , and 4 layers labeled. Raman spectra on the same sheet show clear $G$ and $2 D$ bands with a peak intensity ratio

${ }^{a)}$ Electronic mail: xhong@phys.psu.edu.
$I(2 \mathrm{D}) / I(\mathrm{G})=1.3$ for single layers and 0.65 for bilayers. The 2D band becomes broader and progressively weaker in thicker flakes. Stand-alone thin flakes $(n=2-15)$ are fabricated into FETs using standard lithography procedures. ${ }^{8}$ Transport measurements are performed using lock-in techniques in a pumped ${ }^{4} \mathrm{He}$ cryostat. The carrier density $n$ is determined via Hall measurements, from which we deduce the gating efficiency of the device and the corresponding dielectric constant $\kappa$ of the PZT. ${ }^{8}$ For devices reported here, $\kappa$ varies from $30-100$.

In Fig. 2, we show the sheet resistance, $\rho$, as a function of the backgate voltage, $V_{\mathrm{g}}$, of a 7-LG device at $300 \mathrm{~K}$. The gating efficiency of this device is $1.35 \times 10^{12} \mathrm{~cm}^{-2} / V_{\mathrm{g}}(\mathrm{V})$, corresponding to $\kappa \approx 100 . \rho\left(V_{\mathrm{g}}\right)$ exhibits distinct behaviors at low and high $V_{\mathrm{g}}$. When the gate sweep is limited to $\left|V_{\mathrm{g}}\right|$ $<2 \mathrm{~V}$ (black curve), the carrier density and $\rho\left(V_{\mathrm{g}}\right)$ follow conventional field effect modulation, and the forward and backward sweeps reproduce one another. $\rho\left(V_{\mathrm{g}}\right)$ reaches a maximum at the charge neutrality point at $V_{\mathrm{g}}=0.17 \mathrm{~V}$. In this regime, we observe a Hall mobility $\mu_{\mathrm{H}}$ up to $70000 \mathrm{~cm}^{2} / \mathrm{V} \mathrm{s}^{8}$. The small initial doping of $\sim 2$
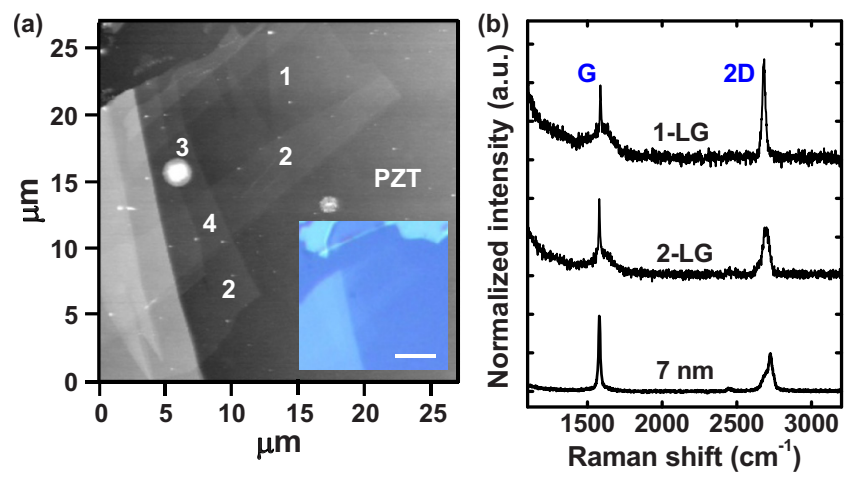

FIG. 1. (Color online) (a) AFM image of a multilayer graphene sheet on a $300 \mathrm{~nm}$ PZT film. The layer numbers are determined by height measurements and marked in the figure. Inset: optical image of the same sheet. Scale bar: $10 \mu \mathrm{m}$. (b) Raman spectra on different parts of the sheet shown in (a) normalized to the $\mathrm{G}$ peak intensity. The increasing background at low wave numbers and the small broad peak centered at $1615 \mathrm{~cm}^{-1}$ are from the PZT substrate. 


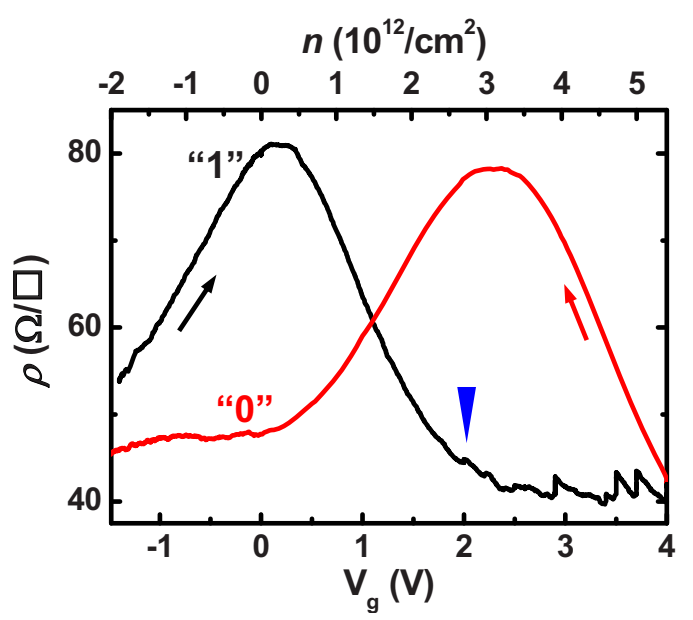

FIG. 2. (Color online) $\rho\left(V_{\mathrm{g}}\right)$ on a 7-LG FET at $300 \mathrm{~K} . \rho\left(V_{\mathrm{g}}\right)$ reaches a maximum at the charge neutrality point. Arrows indicate the sweeping direction of $V_{\mathrm{g}}$. The top axis labels the corresponding carrier density scale. $\left(V_{\mathrm{g}}=2 \mathrm{~V}\right.$ marked by the blue triangle.) The "0" and "1" labels illustrate the "off" and "on" states for a memory operation.

$\times 10^{11} / \mathrm{cm}^{2}$ indicates that the large remnant polarization of PZT has been mostly screened by charged adsorbates and surface reconstruction prior to graphene exfoliation. ${ }^{12,13}$

At $V_{\mathrm{g}}>2 \mathrm{~V}, \rho\left(V_{\mathrm{g}}\right)$ becomes hysteretic, with the backward sweeping curve shifted to the right of the forward sweeping curve (Fig. 2). The onset of the hysteresis is accompanied by a saturation in $\rho\left(V_{\mathrm{g}}\right)$, and a similar trend is observed in $n\left(V_{\mathrm{g}}\right)$. The onset $V_{\mathrm{g}}$ of the hysteresis upon forward sweep roughly coincides with the charge neutrality point in backward sweep, and vice versa. The shift between the forward and backward $\rho\left(V_{\mathrm{g}}\right)$ corresponds to $\Delta n=2.7$ $\times 10^{12} / \mathrm{cm}^{2}$. Similar hysteresis is observed on four PZTgated $n$-LG devices fabricated on three different PZT films, ${ }^{10}$ regardless of the thickness of the graphene sheet (2-15 layers), its carrier mobility $\left(16000-140000 \mathrm{~cm}^{2} / \mathrm{V} \mathrm{s}\right)$ and the dielectric constant (30-100) of the PZT.

Several features of the hysteresis rule out ferroelectric polarization switching as its origin. First, the direction of the hysteresis is opposite to that expected from carrier density change induced by the polarization reversal of PZT. We thus refer to this behavior as "antihysteresis." Second, $\Delta n$ is only $1 \%$ of the nominal $2 \mathrm{D}$ charge density corresponding to the polarization of PZT $\left(\sim 3 \times 10^{14} / \mathrm{cm}^{2}\right)$. Third, the hysteresis occurs at $V_{\mathrm{g}}$ smaller than the coercive voltage of PZT necessary to reverse the polarization.

The observed antihysteresis in $\rho\left(V_{\mathrm{g}}\right)$ is reproducible and characterized by long relaxation times. In Fig. 2, the black curve is the stable state at lower $V_{\mathrm{g}}$, while the red curve is more stable at higher $V_{\mathrm{g}}$. If we sweep $V_{\mathrm{g}}$ from $0 \mathrm{~V}$ following the black curve and pause at $V_{\mathrm{g}}=2 \mathrm{~V}$, the system slowly relaxes to the red curve resistance $\rho_{\text {red. }}$. The normalized resistance difference $\delta \rho(t) / \delta \rho(0)$ follows an exponential time dependence given by $\exp (-t / \tau)$ as shown in Fig. 3(a). Here $\delta \rho=\rho(t)-\rho_{\text {red }}$ is the time-dependent $\rho(t)$ relative to $\rho_{\text {red }}$ at $V_{\mathrm{g}}=2 \mathrm{~V}$, and $\tau$ the relaxation time constant. $\tau$ increases from $6 \mathrm{~h}$ at $300 \mathrm{~K}$ to 80 days at $77 \mathrm{~K}$ [Fig. 3(b)]. The $T$-dependence of the relaxation rate, $1 / \tau$, can be roughly described by
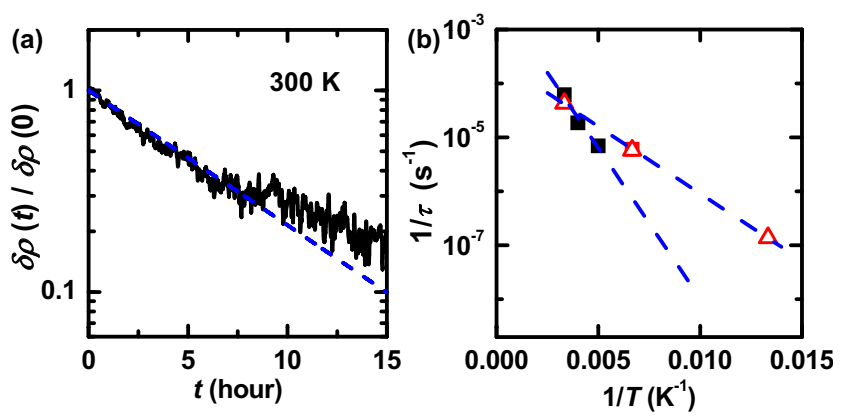

FIG. 3. (Color online) (a) Time-dependent relaxation from the metastable resistance state (the forward sweep curve in Fig. 2) toward the stable state (the backward sweep curve) at $V_{\mathrm{g}}=2 \mathrm{~V}$. The blue dashed line is an exponential fit to $\exp (-t / \tau)$ with $\tau=6 \mathrm{~h}$. (b) Arrhenius plot of $1 / \tau$ vs temperature from measurements in (a) (solid squares) and similar measurements on another 15-LG device (open triangles). Blue dashed lines are fittings to Eq. (1) with $E_{b}=50$ (upper line) and $110 \mathrm{meV}$ (lower line).

$$
\frac{1}{\tau} \sim \exp \left[-\frac{\Delta E_{b}}{k_{B} T}\right],
$$

suggesting a thermally activated relaxation process between two metastable states separated by an activation barrier. Figure 3(b) shows the Arrhenius plot of $1 / \tau$ in two devices. The barrier height $\Delta E_{b}$ is estimated to be $50-110 \mathrm{meV}$ by fitting to Eq. (1).

Such antihysteresis behavior was previously observed in carbon nanotube FETs gated by $\mathrm{SiO}_{2}$ and epitaxial ferroelectric $\mathrm{BaTiO}_{3}$ films. ${ }^{6,14,15}$ Several explanations involving oxide charge trapping, surface charge trapping via silanol groups, or the polarization of surface bound water molecules have been proposed. ${ }^{16-18}$ Although the exact mechanism has yet to be clarified, studies in Ref. 15 point to a crucial role played by interfacial water molecules.

In our PZT-gated devices, charged adsorbates are present on the surface of PZT to screen its polarization prior to the exfoliation of graphene. As a likely candidate in the ambient, water is known to have two metastable forms when chemisorbed on the surface of transition metal oxides, such as $\mathrm{TiO}_{2}, \mathrm{VO}_{2}$, and $\mathrm{BaTiO}_{3}$. It either maintains its molecular form or, in the presence of defects or uncompensated charges, dissociates into $\mathrm{H}^{+}$and $\mathrm{OH}^{-}{ }^{19-21}$ The balance between the dissociation and recombination processes

$$
\mathrm{H}_{2} \mathrm{O} \Leftrightarrow \mathrm{H}^{+}+\mathrm{OH}^{-},
$$

is influenced by the geometry of the lattice and external electric fields. ${ }^{20,21}$ It has also been shown that $\mathrm{OH}^{-}$chemisorbs on the $\mathrm{Pb}^{2+}$ sublattice of ultrathin $\mathrm{PbTiO}_{3}$ to screen its polarization, with a binding energy of $\sim 200 \mathrm{meV}{ }^{12}$

Given the similarity between our observations and those reported for carbon nanotube devices, and the surface dynamics of water adsorbed on transition metal oxides, we propose to use the dissociation-recombination of water molecules adsorbed at the graphene-PZT interface to account for the antihysteresis seen in our devices. In this model, $\mathrm{OH}^{-}$ and $\mathrm{H}^{+}$dissociated from chemisorbed water, as well as molecular $\mathrm{H}_{2} \mathrm{O}$, are likely to be present on the PZT surface to screen its polarization prior to the exfoliation of graphene. A positive $V_{\mathrm{g}}$ applied to PZT reduces $P$ (originally uniformly polarized) and results in over-screening from the adsorbates. The balance of Eq. (2) is thus driven toward recombination. This process is thermally activated [Eq. (1)] due to the for- 
mation of a hydrogen bond between the $\mathrm{H}^{+}$ion and the surface anion $\mathrm{O}^{2-}$, which acts as the activation barrier. The estimated barrier height $\Delta E_{b} \sim 50-110 \mathrm{meV}$ is consistent with the binding energy of a weak hydrogen bond on a transition metal oxide surface. ${ }^{12,20,21}$ Conversely, as $V_{\mathrm{g}}$ decreases, $P$ increases and results in under-screening from the adsorbates. Dissociation of water is then favored to provide additional screening, which also needs to overcome $\Delta E_{b}$ on the order of a hydrogen bond. Both processes attempt to screen the polarization change in PZT, which in turn prevents charge injection into graphene and leads to the observed antihysteresis in resistance and carrier density. This model naturally explains the remarkable similarity among devices fabricated on films with a wide range of $\kappa$, since the relevant energy scales are determined by the surface chemistry of water on PZT, rather than PZT's bulk properties.

This robust hysteresis can potentially be used to implement a memory device, where the " 0 " and " 1 " states are represented by the low (red) and high (black) resistance states, as shown in Fig. 2. The high/low resistance ratio is 2 for the $7-\mathrm{LG}$ and reaches 3.5 in a 2 -LG device. ${ }^{10}$ The high- $\kappa$ nature of PZT (up to 100) and the superb room temperature carrier mobility in these devices (up to $70000 \mathrm{~cm}^{2} / \mathrm{V} \mathrm{s}$ ) should enable reading and writing operations at small gate voltages and exceedingly high frequencies. To achieve the retention time necessary for a practical nonvolatile memory device, controlled adsorbates with higher binding energies may be required.

In conclusion, we observe an unusual resistance hysteresis in high-mobility $n$-layer graphene FETs fabricated on epitaxial PZT thin films. We attribute its origin to the dynamic dissociation/recombination of water molecules chemisorbed at the PZT surface. The reproducibility of the hysteresis, combined with excellent carrier mobility, provide a viable pathway of implementing memory functions in graphene-oxide hybrid devices.

We are grateful to V. Crespi, P. Eklund, V. Henrich, P. Paruch, and X. Pan for helpful discussions and S.-H. Cheng and B. Wang for technical assistance. Work at Penn State is supported by NSF NIRT under Grant No. ECS-0609243 and
NSF CAREER under Grant No. DMR-0748604. Fabrication of samples at Yale is supported by NSF MRSEC under Grant No. DMR-0520495, NSF under Grant No. DMR-0705799, ONR under Grant No. N00014-09-1-0081, and NRI. The authors also acknowledge use of NSF NNIN facilities at PSU.

${ }^{1}$ A. K. Geim, Science 324, 1530 (2009).

${ }^{2}$ Y. M. Lin, H. Y. Chiu, K. A. Jenkins, D. B. Farmer, P. Avouris, and A. Valdes-Garcia, IEEE Electron Device Lett. 31, 68 (2010).

${ }^{3}$ N. Tombros, C. Jozsa, M. Popinciuc, H. T. Jonkman, and B. J. van Wees, Nature (London) 448, 571 (2007).

${ }^{4}$ W. H. Wang, K. Pi, Y. Li, Y. F. Chiang, P. Wei, J. Shi, and R. K. Kawakami, Phys. Rev. B 77, 020402 (2008).

${ }^{5}$ B. Lei, C. Li, D. Zhang, Q. Zhou, K. Shung, and C. Zhou, Appl. Phys. Lett. 84, 4553 (2004).

${ }^{6}$ P. Paruch, A.-B. Posadas, M. Dawber, C. H. Ahn, and P. L. McEuen, Appl. Phys. Lett. 93, 132901 (2008).

${ }^{7}$ Y. Zheng, G.-X. Ni, C.-T. Toh, M.-G. Zeng, S.-T. Chen, K. Yao, and B. Özyilmaz, Appl. Phys. Lett. 94, 163505 (2009).

${ }^{8}$ X. Hong, A. Posadas, K. Zou, C. H. Ahn, and J. Zhu, Phys. Rev. Lett. 102, 136808 (2009).

${ }^{9}$ X. Hong, A. Posadas, A. Lin, and C. H. Ahn, Phys. Rev. B 68, 134415 (2003).

${ }^{10}$ See supplementary material at http://dx.doi.org/10.1063/1.3467450 for characterization details of the PZT films and resistance hysteresis observed on a $2-L G$ device.

${ }^{11}$ A. Lin, X. Hong, V. Wood, A. A. Verevkin, C. H. Ahn, R. A. McKee, F. J. Walker, and E. D. Specht, Appl. Phys. Lett. 78, 2034 (2001).

${ }^{12}$ D. D. Fong, A. M. Kolpak, J. A. Eastman, S. K. Streiffer, P. H. Fuoss, G. B. Stephenson, C. Thompson, D. M. Kim, K. J. Choi, C. B. Eom, I. Grinberg, and A. M. Rappe, Phys. Rev. Lett. 96, 127601 (2006).

${ }^{13}$ B. Meyer and D. Vanderbilt, Phys. Rev. B 63, 205426 (2001).

${ }^{14}$ M. Fuhrer, B. Kim, T. Durkop, and T. Brintlinger, Nano Lett. 2, 755 (2002).

${ }^{15}$ W. Kim, A. Javey, O. Vermesh, O. Wang, Y. Li, and H. Dai, Nano Lett. 3, 193 (2003).

${ }^{16}$ J. S. Lee, S. Ryu, K. Yoo, I. S. Choi, W. S. Yun, and J. Kim, J. Phys. Chem. C 111, 12504 (2007).

${ }^{17}$ A. Robert-Peillard and S. Rotkin, IEEE Trans. Nanotechnol. 4, 284 (2005).

${ }^{18}$ A. Guo, Y. Fu, C. Wang, L. Guan, J. Liu, Z. Shi, Z. Gu, R. Huang, and X. Zhang, Nanotechnology 18, 125206 (2007).

${ }^{19}$ F. Peter, K. Szot, R. Waser, B. Reichenberg, S. Tiedke, and J. Szade, Appl. Phys. Lett. 85, 2896 (2004).

${ }^{20}$ M. A. Henderson, Langmuir 12, 5093 (1996).

${ }^{21}$ M. A. Henderson, Surf. Sci. Rep. 46, 1 (2002). 